\title{
Fulde-Ferrell-Larkin-Ovchinnikov correlation and free fluids in the one-dimensional attractive Hubbard model
}

\author{
Song Cheng, ${ }^{1,2,3}$ Yi-Cong Yu, ${ }^{1,2}$ M. T. Batchelor, ${ }^{3,4,5}$ and Xi-Wen Guan ${ }^{1,3,6, *}$ \\ ${ }^{1}$ State Key Laboratory of Magnetic Resonance and Atomic and Molecular Physics, Wuhan Institute of Physics and Mathematics, \\ Chinese Academy of Sciences, Wuhan 430071, China \\ ${ }^{2}$ University of Chinese Academy of Sciences, Beijing 100049, China \\ ${ }^{3}$ Department of Theoretical Physics, Research School of Physics and Engineering, Australian National University, \\ Canberra ACT 0200, Australia \\ ${ }^{4}$ Centre for Modern Physics, Chongqing University, Chongqing 400044, China \\ ${ }^{5}$ Mathematical Sciences Institute, Australian National University, Canberra ACT 0200, Australia \\ ${ }^{6}$ Center for Cold Atom Physics, Chinese Academy of Sciences, Wuhan 430071, China
}

(Received 24 August 2017; revised manuscript received 28 December 2017; published 26 March 2018)

\begin{abstract}
In this Rapid Communication, we show that low-energy macroscopic properties of the one-dimensional (1D) attractive Hubbard model exhibit two fluids of bound pairs and of unpaired fermions. Using the thermodynamic Bethe ansatz equations of the model, we first determine the low-temperature phase diagram and analytically calculate the Fulde-Ferrell-Larkin-Ovchinnikov (FFLO) pairing correlation function for the partially polarized phase. We then show that for such an FFLO-like state in the low-density regime the effective chemical potentials of bound pairs and unpaired fermions behave like two free fluids. Consequently, the susceptibility, compressibility, and specific heat obey simple additivity rules, indicating the "free" particle nature of interacting fermions on a 1D lattice. In contrast to the continuum Fermi gases, the correlation critical exponents and thermodynamics of the attractive Hubbard model essentially depend on two lattice interacting parameters. Finally, we study scaling functions, the Wilson ratio and susceptibility, which provide universal macroscopic properties and dimensionless constants of interacting fermions at low energy.
\end{abstract}

DOI: 10.1103/PhysRevB.97.121111

The notion of Landau quasiparticles gives rise to the Fermi liquid theory successfully used for describing properties of a large variety of systems, such as Fermi liquid ${ }^{3} \mathrm{He}$ and electrons in metals [1]. In contrast, it is generally accepted that Fermi liquid theory is not applicable in $1 \mathrm{D}$, where the description of the low-energy physics of strongly correlated electrons, spins, bosonic and fermionic atoms relies on the TomonagaLuttinger liquid (TLL) theory [2]. Such an understanding of the TLL in 1D is based on collective excitations, which are significantly different from Landau quasiparticles in higher dimensions. However, concerning macroscopic properties, there are many universal properties/quantities that are common for both 2D/3D and 1D systems [3-6].

The 1D repulsive Fermi-Hubbard model describing interacting fermions on a lattice provides a paradigm for understanding many-body physics, including spin-charge separation, fractional excitations, quantum dynamics of spinons, a Mott insulating phase, and magnetism [7]. Very recently, ultracold atoms trapped in optical lattices [8-12] offer promising opportunities to test such fundamental concepts [13]. In contrast, the 1D attractive Fermi-Hubbard model [14-20] is a notoriously difficult problem due to the complicated bound states of multiparticles and multispins on lattices. Despite there being a mapping by Shiba transformations between the repulsive and attractive regions of the Hubbard model [7],

*xiwen.guan@anu.edu.au such a mapping cannot be used for a study of the lowenergy themodynamics of the attractive Hubbard model due to the different cutoff processes in terms of such multispin and multicharge bound states. Of central importance to this attractive Hubbard model is the understanding of quantum correlations of charge bound states, for example, the FuldeFerrell-Larkin-Ovchinnikov (FFLO) like pairing $[21,22]$ on a 1D lattice [23-25]. In the expansion dynamics of the FFLO state in 1D [26], a nature of two fluids of bound pairs and free fermions was indicated.

In this Rapid Communication, building on the thermodynamic Bethe ansatz (TBA) equations of the attractive Hubbard model, we analytically obtain the FFLO pairing correlation and the universal two free quantum fluids of the FFLO-like state, where the lattice effects are seen to drive the system differently to the continuous Fermi gas [27-32], see Fig. 1. More detailed studies of this model will be presented elsewhere $[33,34]$.

The Bethe ansatz solution. The 1D single-band Hubbard model is described by the Hamiltonian [7]

$$
\begin{aligned}
H= & -\sum_{j=1, a=\uparrow, \downarrow}^{L}\left(c_{j, a}^{\dagger} c_{j+1, a}+\text { H.c. }\right) \\
& +u \sum_{j=1}^{L}\left(2 n_{j, \uparrow}-1\right)\left(2 n_{j, \downarrow}-1\right),
\end{aligned}
$$

where $c_{j, a}^{\dagger}$ and $c_{j, a}$ are the creation and annihilation operators of electrons (fermionic atoms) with spin $a$ (internal degrees of 


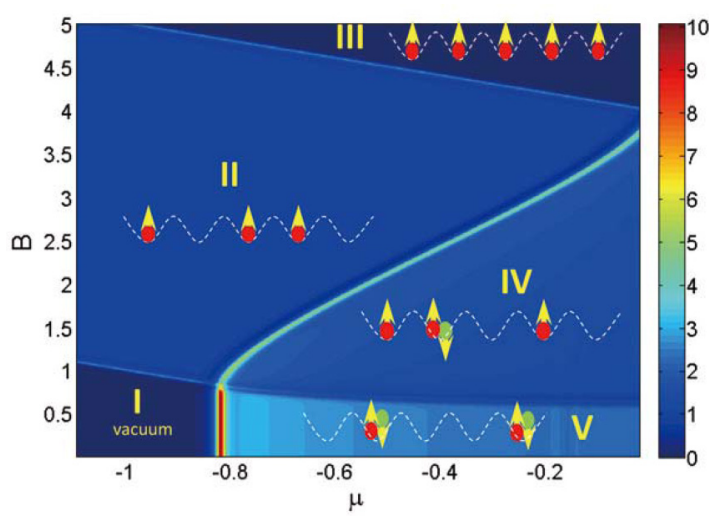

FIG. 1. Low-temperature phase diagram determined by the contour plot of the Wilson ratio $R_{\mathrm{W}}^{\kappa}(5)$ calculated from the TBA equations (1)-(3) for the 1D attractive Hubbard model in the $\mu-B$ plane at $T=0.01$ and $u=-1$. Here the setting is chosen for better visibility. Up (down) spins are represented by red (green) balls. Different values of this ratio uniquely present five quantum phases. Sudden enhancement of the ratio in the vicinity of critical lines marks the phase boundaries between different phases, see text.

freedom) ( $a=\uparrow$ or $a=\downarrow)$ at site $j$ on a 1D lattice with length $L$. They satisfy the anticommutation relations $\left\{c_{j, a}, c_{k, b}\right\}=$ $\left\{c_{j, a}^{\dagger}, c_{k, b}^{\dagger}\right\}=0$ and $\left\{c_{j, a}, c_{k, b}^{\dagger}\right\}=\delta_{j k} \delta_{a b}$. Meanwhile, $n_{j, a}=$ $c_{j, a}^{\dagger} c_{j, a}$ is the density operator, $n_{e}=\frac{1}{L} \sum_{j=1}^{L} \sum_{a} n_{j, a}$ is the total fermion number per lattice site and $u$ is the dimensionless interaction strength between particles $(u>0$ for repulsion and $u<0$ for attraction).

In 1968, Lieb and Wu [35] derived the Bethe ansatz (BA) equations for the 1D Hubbard model by means of Bethe's hypothesis [36]. Takahashi [37,38] discovered the solutions of the BA equations, which in general are classified as real quasimomenta $k, k-\Lambda$ strings and complex spin rapidities of $\Lambda$ strings, see Ref. [39]. These roots respectively count for the quasimomenta of the single fermions, bound states of different lengths of fermions and bound states of magnons with different lengths. At high energy or momentum, such bound states can coexist. Building on Takahashi's string hypothesis, we obtain the TBA equations for the 1D attractive Hubbard model [33]:

$$
\begin{gathered}
\varepsilon(k)=g_{0}(k)-\sum_{n=1}^{\infty} a_{n} *\left(F\left[\varepsilon_{n}^{\prime}\right]-F\left[\varepsilon_{n}\right]\right)(k), \\
\varepsilon_{n}(\Lambda)=2 n B-a_{n}^{t} * F[\varepsilon](\Lambda)-\sum_{m=1}^{\infty} A_{n m} * F\left[\varepsilon_{m}\right](\Lambda), \\
\varepsilon_{n}^{\prime}(\Lambda)=g_{n}(\Lambda)-a_{n}^{t} * F[\varepsilon](\Lambda)-\sum_{m=1}^{\infty} A_{n m} * F\left[\varepsilon_{m}^{\prime}\right](\Lambda)
\end{gathered}
$$

with the notation $F[x](y)=-T \ln [1+\exp (-x(y) / T)]$ and $n=1, \ldots, \infty$. The kernel function $a_{n}(x)=\frac{1}{2 \pi} \frac{2 n|u|}{(n|u|)^{2}+x^{2}}$. The driving terms are $g_{0}(y)=-2 \cos y-\mu-2 u-B$ and $g_{n}(y)=-4 \operatorname{Re} \sqrt{1-(y+\mathrm{i} n|u|)^{2}}-n(2 \mu+4 u)$. In the above equations, we denoted the convolutions $a_{n} * F[x](k)=\int_{-\infty}^{\infty}$ $\mathrm{d} y a_{n}(k-y) F[x(y)]$ and $a_{n}^{t} * F[x](\Lambda)=\int_{-\pi}^{\pi} \mathrm{d} y \cos y a_{n}$ $(\sin y-\Lambda) F[x(y)]$. The functions $\varepsilon, \varepsilon_{m}^{\prime}$, and $\varepsilon_{n}$ stand for the dressed energies for unpaired fermions, bound states of $2 m$ fermions (the $k-\Lambda$ strings) and length- $n$ spin strings of magnons, respectively. The function $A_{n m}(x)$ is given in Ref. [33].

It is particularly important to observe that the longer $k-\Lambda$ strings are involved in the thermodynamics as temperature increases [40]. The free energy per site is thus given by

$f=u+\int_{-\pi}^{\pi} \frac{\mathrm{d} k}{2 \pi} F[\varepsilon](k)+\sum_{n=1}^{\infty} \int_{-\infty}^{\infty} \frac{\mathrm{d} \Lambda}{2 \pi} \xi_{n}(\Lambda) F\left[\varepsilon_{n}^{\prime}\right](\Lambda)$

with $\xi_{n}(\Lambda)=\int_{-\pi}^{\pi} \mathrm{d} k a_{n}(\Lambda-\sin k)$. We also observe that in the dilute limit, $u \rightarrow 0, n_{e} \rightarrow 0$ with $n_{e} /|u|$ constant [14], the TBA equations (1)-(3) reduce to those of the Gaudin-Yang model $[7,29,39]$. We note that the Shiba transformation between the repulsive and attractive regions of the Hubbard model does not help to obtain universal low-energy physics from the TBA equations. This is mainly because the cutoff processes regarding the above spin and charge bound states are quite different [33], unlike the case of the ground state [20]. As we shall see, in the attractive regime, the low-energy physics of the model is no longer described by the spin-charge separated theory, rather it is described by the FFLO-like quantum liquids of pairs and single fermions.

Quantum phase diagram and Wilson ratio. In contrast to the repulsive case, the ground state of the attractive Hubbard model has charge bound states, i.e., length- $1 k$ - $\Lambda$ strings, forming a lattice version of the FFLO state. The quantum phases and phase diagram at $T=0$ can be directly determined from the TBA equations (1)-(3) in the limit $T \rightarrow 0$, which are called the dressed energy equations [33]. The dressed energy equations determine five quantum phases in the $\mu-B$ plane: vacuum I, fully-polarized phase II, half-filled phase III, FFLO-like state IV, and fully-paired state V, see Fig. 1. The zero-temperature phase boundaries can also be determined by the Shiba transformation [7].

Here we show that the Wilson ratio, namely, the dimensionless ratio of the compressibility $\kappa$ and the specific heat divided by the temperature $T$,

$$
R_{\mathrm{W}}^{\kappa}=\frac{\pi^{2} k_{\mathrm{B}}^{2}}{3} \frac{\kappa}{C_{v} / T},
$$

provides a convenient way for revealing the full phase diagram at low temperatures, see Fig. 1 . In the above, $k_{\mathrm{B}}$ is Boltzmann's constant. This ratio can be directly calculated from the finite temperature TBA equations (1)-(3) with a suitable spin and charge bound state cutoff process, see Ref. [33]. We find that the ratio $R_{\mathrm{W}}^{\kappa}$ is capable of distinguishing all phases of quantum states, including the FFLO-like state in the phase diagram Fig. 1. We observe that an enhancement of this ratio occurs near a phase transition. It gives a finite value at the critical point unlike the divergent values of compressibility and susceptibility for $T \rightarrow 0$. Indeed, the phase boundaries determined by the Wilson ratio (5) coincide with the ones determined by the dressed energy equations at $T=0$.

The phases IV and V in Fig. 1 reveal significant features, namely, the quasi-long-range order and free-fermion quantum criticality. A constant Wilson ratio implies that the two types of fluctuations are on an equal footing, regardless of the microscopic details of the underlying many-body systems. 


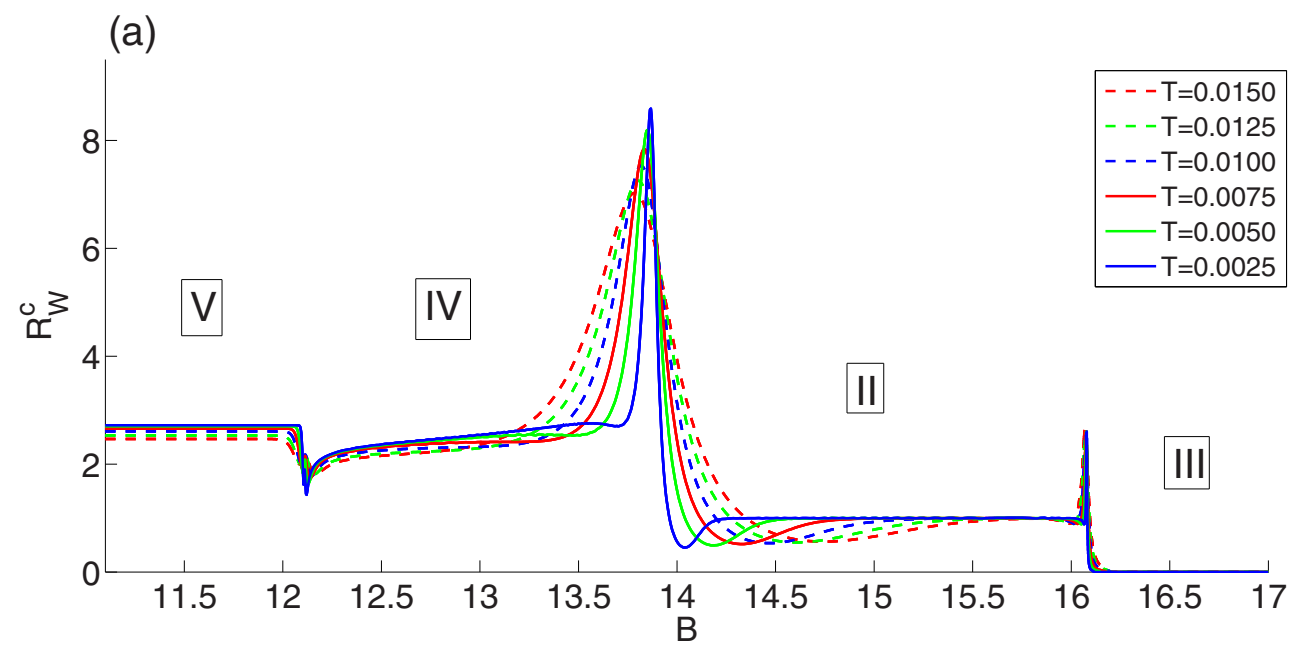

(b)

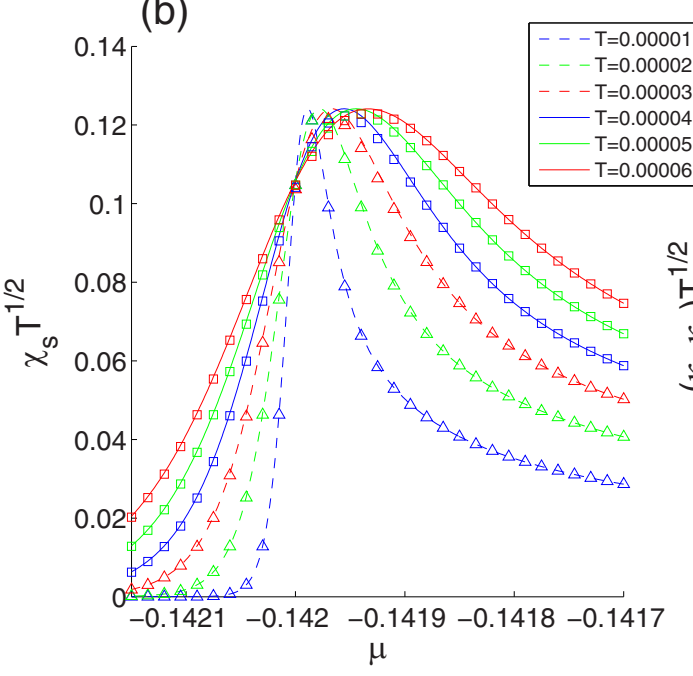

(c)

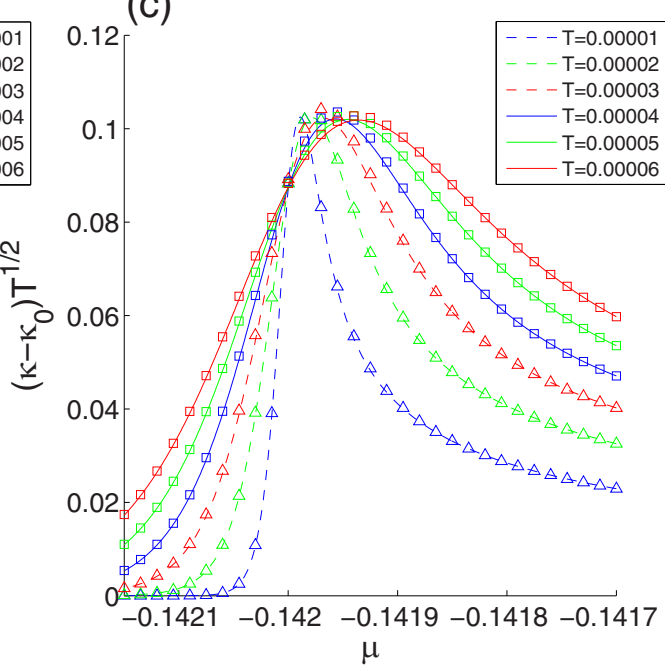

FIG. 2. (a) Wilson Ratio $R_{\mathrm{W}}^{\kappa_{c}}$ vs magnetic field for fixed $\mu=-0.08$ and $u=-7$ in the strong-coupling regime. The sharp peaks at phase transitions distinguish different quantum phases V, IV, II, and III, respectively. The constant values of the ratio show Fermi liquid nature in these phases. (b) and (c) show the scaling invariant behavior of the susceptibility and compressibility for a fixed $B=12.142$. The numerical TBA results (lines) perfectly confirm the analytical scaling functions (6) and (7) (symbols).

Regarding the sudden change of the Wilson ratio near a phase transition, we observe that the particle number and energy fluctuations become temperature dependent, see Fig. 2(a). At the critical point, the vanishing of the Fermi points, i.e., $\varepsilon_{1}(0)=0$ and $\varepsilon_{1}^{\prime}(0)=0$, in the Fermi sea of pairs and of unpaired fermions leads to a universality class of quantum criticality. In the critical regime, the scaling functions of thermodynamic properties can be cast into universal forms. From the TBA equations (1)-(3), we obtain the scaling functions of compressibility and susceptibility:

$$
\begin{aligned}
& \kappa(\mu, B, T)=\kappa_{0}(\mu, B)+T^{\frac{d}{z}+1-\frac{2}{v z}} \lambda_{\kappa} \mathcal{F}\left(\frac{\mu-\mu_{c}}{T^{1 / v z}}\right), \\
& \chi(\mu, B, T)=\chi_{0}(\mu, B)+T^{\frac{d}{z}+1-\frac{2}{v z}} \lambda_{\chi} \mathcal{K}\left(\frac{\mu-\mu_{c}}{T^{1 / v z}}\right) .
\end{aligned}
$$

Here the scaling functions $\mathcal{F}(x)=\mathcal{K}(x)=\mathrm{Li}_{-1 / 2}(x)$ indicate a free-fermion criticality classified by the dynamical critical exponents $z=2$ and correlation critical exponent $v=1 / 2$, see [33]. The terms $\kappa_{0}$ and $\chi_{0}$ are the regular part and the factors $\lambda_{\kappa, \chi}$ are phase dependent constants. Figures 2(b) and 2(c) show such universal scaling behavior of the susceptibility and compressibility across the phase boundary (V,IV). Similar scaling invariant behavior occurs whenever the model parameters are driven across the phase boundaries in Fig. 1.

FFLO correlation. For the fully paired state $\mathrm{V}$, the pairing correlation length is larger than the average interparticle spacing. In this phase, the single-particle Green's function decays exponentially, whereas the singlet pair correlation function decays as a power of distance [15]. However, once the external field exceeds the critical line between phases IV and V, the Cooper pairs start to break apart. Thus both of these correlation functions decay as a power of distance, indicating a quasi-long-range correlation. In the phase IV, Cooper pairs and excess fermions form a 1D analog of the FFLO pairing-like state [24,25]. However, analytical result for the FFLO pairing correlations for the Hubbard model is still lacking. For obtaining a universal form of the FFLO-like correlation function, we first focus on the case of low density $n_{e} \ll 1$ and low energy. In the FFLO-like phase IV, the spin 
wave bound states ferromagnetically couple to the Fermi sea of the unpaired fermions. Thus the spin wave fluctuations can be ignored at low temperatures due to this ferromagnetic nature. Then we simplify the TBA equations (1)-(3) as [33]

$$
\begin{gathered}
\varepsilon(k) \approx k^{2}-\mu_{1}-a_{1} * F\left[\varepsilon_{1}^{\prime}\right](k), \\
\varepsilon_{1}^{\prime}(\Lambda) \approx \alpha_{1}\left(\Lambda^{2}-\mu_{2}\right)-a_{1} * F[\varepsilon](\Lambda)-a_{2} * F\left[\varepsilon_{1}^{\prime}\right](\Lambda) .
\end{gathered}
$$

The free energy (4) reduces to $f \approx u+\int_{-\pi}^{\pi} \frac{\mathrm{d} k}{2 \pi} F[\varepsilon](k)+$ $\int_{-\infty}^{\infty} \frac{\mathrm{d} \Lambda}{2 \pi} \beta_{1} F\left[\varepsilon_{1}^{\prime}\right](\Lambda)$ [41]. In this new set of TBA equations (8) and (9), we have introduced two effective chemical potentials,

$$
\begin{aligned}
& \mu_{1}=\mu-2|u|+B+2, \\
& \mu_{2}=\frac{1}{\alpha_{1}}\left[2 \mu+4\left(\sqrt{u^{2}+1}-|u|\right)\right],
\end{aligned}
$$

for understanding the FFLO correlation and free-fermion nature of the attractive Hubbard model. In the above equations, the parameters $\alpha_{n}$ and $\beta_{n}$ reflect the interacting effect of the length- $n k-\Lambda$ bound states on a lattice. They are given by

$$
\begin{aligned}
& \alpha_{n}=\int_{-\pi}^{\pi} \mathrm{d} k \cos ^{2} k \frac{2 n|u| \cos ^{2} k\left(n^{2} u^{2}-3 \sin ^{2} k\right)}{\pi\left(n^{2} u^{2}+\sin ^{2} k\right)^{3}}, \\
& \beta_{n}=\int_{-\pi}^{\pi} \mathrm{d} k a_{n}(\sin k) .
\end{aligned}
$$

At low-energy physics, only length- $1 k-\Lambda$ strings are involved. In this region, the lattice parameters $\alpha_{1}$ and $\beta_{1}$ approach 2 when $u$ tends to zero. However, for large $|u|$, the band of pairs becomes flat [33]. The TBA equations (8) and (9) are reminiscent of the "feedback interaction" equation in the Landau-Fermi liquid theory $[3,42]$. The driving term in (9) can be expressed as $\frac{\hbar^{2}}{2 m} \alpha_{1}\left(k^{2}-\mu_{2}\right)=\frac{p_{0}^{2} \alpha_{1}}{2 m}-\frac{\hbar^{2}}{2 m} \alpha_{1} \mu_{2}$ with $2 m=\hbar=1$, which is the first-order coefficient describing the excitation energy of a single bound pair. The lattice parameter $\alpha_{n}$ characterizes the effective mass of length- $n k-\Lambda$ strings (bound state of $2 n$ atoms on a lattice).

In light of the conformal field theory approach [43-45] and using the TBA equations (8) and (9), we calculate the asymptotic form of the FFLO correlation function of the attractive Hubbard model in the low-density region [46]:

$$
\begin{aligned}
G_{p}(x, t)= & \left\langle\Psi_{\uparrow}^{\dagger}(x, t) \Psi_{\downarrow}^{\dagger}(x, t) \Psi_{\uparrow}(0,0) \Psi_{\downarrow}(0,0)\right\rangle \\
\approx & A_{p, 1} \frac{\cos \left(\pi\left(n_{\uparrow}-n_{\downarrow}\right) x\right)}{\left|x+\mathrm{i} v_{u} t\right|^{2 \theta_{1}}\left|x+\mathrm{i} v_{b} t\right|^{2 \theta_{2}}} \\
& +A_{p, 2} \frac{\cos \left(\pi\left(n_{\uparrow}-3 n_{\downarrow}\right) x\right)}{\left|x+\mathrm{i} v_{u} t\right|^{2 \theta_{3}}\left|x+\mathrm{i} v_{b} t\right|^{2 \theta_{4}}},
\end{aligned}
$$

with the exponents $\theta_{1} \approx 1 / 2, \quad \theta_{2} \approx 1 / 2+\frac{n_{2}}{|u| \beta_{1}}, \theta_{3} \approx \frac{1}{2}-$ $\frac{4 n_{2}}{|u| \beta_{1}}$, and $\theta_{4} \approx \frac{5}{2}-\frac{4 n_{1}}{|u|}-\frac{3 n_{2}}{|u| \beta_{1}}$. Here, $n_{2,1}=N_{2,1} / L$ are the dimensionless densities of pairs and unpaired fermions, respectively. The sound velocities are given by $v_{b}=$ $\frac{\sqrt{\alpha_{1}}}{\beta_{1}} \pi n_{2}\left(1+\frac{1}{|u| \beta_{1}}\left(2 n_{1}+n_{2}\right)\right)$ and $v_{u}=\sqrt{2} \pi n_{1}\left(1+\frac{4}{|u|} n_{2}\right)$. In the above equation the coefficients $A_{p, 1}$ and $A_{p, 2}$ are constant factors. In this phase IV, the spatial oscillation in the pairing correlation is a characteristic of the FFLO state, where the imbalance $n_{\uparrow}-n_{\downarrow}$ in the densities of spin-up and spin-down fermions gives rise to a mismatch in Fermi surfaces between both species of fermions. In 1D, the spatial oscillation signature in pair correlation is a consequence of the backscattering for bound pairs and unpaired fermions, see also the results for the Gaudin-Yang model [47]. Here, we observe that the critical exponent $\theta_{2}$ depends essentially on the lattice parameter $\beta_{1}$. So do the critical exponents in other types of correlation functions [34]. The Fourier transform of $G_{P}\left(x, 0^{+}\right)$ gives $\tilde{G}_{p}(k) \sim\left[\operatorname{sign}\left(k-\pi\left(n_{\uparrow}-n_{\downarrow}\right)\right)\right]^{2 s_{p}}\left|k-\pi\left(n_{\uparrow}-n_{\downarrow}\right)\right|^{v_{p}}$ with $2 s_{P} \approx 0$ and $v_{p} \approx n_{2} /\left(|u| \beta_{1}\right)$.

Two free fluids and spin gapped phase. At low temperatures, we find a significant two-fluid nature in phase IV. For the ground state, the energy can be regarded as two TLLs of unpaired fermions and of pairs due to the quasi-long-range correlation. Without losing generality, we consider a physical regime of low density ( $n_{e}$ small), low temperature, and finite strong magnetic field. This region is reachable in cold atoms [13]. In this regime, the chemical potentials for the unpaired fermions and pairs are given explicitly by

$$
\begin{gathered}
\mu_{1}=\pi n_{1}^{2} A_{1}^{2}+\frac{4 \pi^{2} \alpha_{1}}{3 \beta_{1}^{3}|u|} n_{2}^{3} A_{2}^{3}, \\
\mu_{2}=\pi^{2} \frac{n_{2}^{2}}{\beta_{1}^{2}} A_{2}^{2}+\frac{4 \pi^{2}}{3 \alpha_{1}|u|} n_{1}^{3} A_{1}^{3}+\frac{2 \pi^{2}}{3 \beta_{1}^{3}|u|} n_{2}^{3} A_{2}^{3},
\end{gathered}
$$

where $\quad A_{1}=1+\frac{2 n_{2}}{|u|}+\left(\frac{2 n_{2}}{|u|}\right)^{2} \quad$ and $\quad A_{2}=1+\frac{2 n_{1}+n_{2}}{\beta_{1}|u|}+$ $\left(\frac{2 n_{1}+n_{2}}{\beta_{1}|u|}\right)^{2}$ indicate interacting effects among pairs and unpaired fermions like that of the Fermi gas [48]. The effective chemical potential $\mu_{2}$ in the 2D interacting Fermi gases shows a crossover from a Bose-Einstein condensate to a Bardeen-Cooper-Schrieffer superconductor in ultracold fermions [49]. Moreover, from the relations (10), we demonstrate the free-particle nature of two fluids through the additivity rules in compressibility and susceptibility:

$$
\kappa=\kappa_{1}+\frac{2}{\alpha_{1}} \kappa_{2}, \quad \frac{1}{\chi}=\frac{1}{\chi_{1}}+\frac{\alpha_{1}}{2} \frac{1}{\chi_{2}},
$$

where $\kappa_{r}=\left.\left(\partial r n_{r} / \partial \mu_{r}\right)\right|_{B}$ and $\chi_{r}=\left.\left(\partial r n_{r} / \partial \mu_{r}\right)\right|_{n}$ with $r=$ 1,2 for unpaired fermions and pairs, respectively. We see that the effective binding energy $e_{b}=-(2 u+2) n_{1}-4(u+$ $\left.\sqrt{u^{2}+1}\right) n_{2}$ of a bound pair is absorbed into the effective chemical potentials. The compressibility and susceptibility can be explicitly calculated from the chemical potentials (12) and (13) via the relations

$$
\begin{aligned}
& \frac{1}{\kappa_{1}}=\frac{J}{\left(\frac{\partial \mu_{1}}{\partial n_{2}}-\frac{\alpha_{1}}{2} \frac{\partial \mu_{2}}{\partial n_{2}}\right)}, \quad \frac{1}{\kappa_{2}}=-\frac{1}{\alpha_{1}} \frac{J}{\left(\frac{\partial \mu_{1}}{\partial n_{1}}-\frac{\alpha_{1}}{2} \frac{\partial \mu_{2}}{\partial n_{1}}\right)}, \\
& \chi_{1}=\frac{1}{\left(\frac{\partial \mu_{1}}{\partial n_{1}}-\frac{1}{2} \frac{\partial \mu_{1}}{\partial n_{2}}\right)}, \quad \chi_{2}=-\frac{1}{\left(\frac{\partial \mu_{2}}{\partial n_{1}}-\frac{1}{2} \frac{\partial \mu_{2}}{\partial n_{2}}\right)},
\end{aligned}
$$

where the Jacobi determinant $J=-\frac{\alpha_{1}}{2}\left(\frac{\partial \mu_{1}}{\partial n_{1}} \frac{\partial \mu_{2}}{\partial n_{2}}-\frac{\partial \mu_{2}}{\partial n_{1}} \frac{\partial \mu_{1}}{\partial n_{2}}\right)$. The explicit forms are given in Ref. [33]. The additivity rules in the thermodynamic properties reveal a significant free-particle feature in the phase of multiple quantum liquids on a 1D lattice. Furthermore, using the TBA equations (1)-(3) and the BA equations with the length- $1 k-\Lambda$ strings, we show that the specific heat, i.e., a measure of the energy fluctuations, is given 


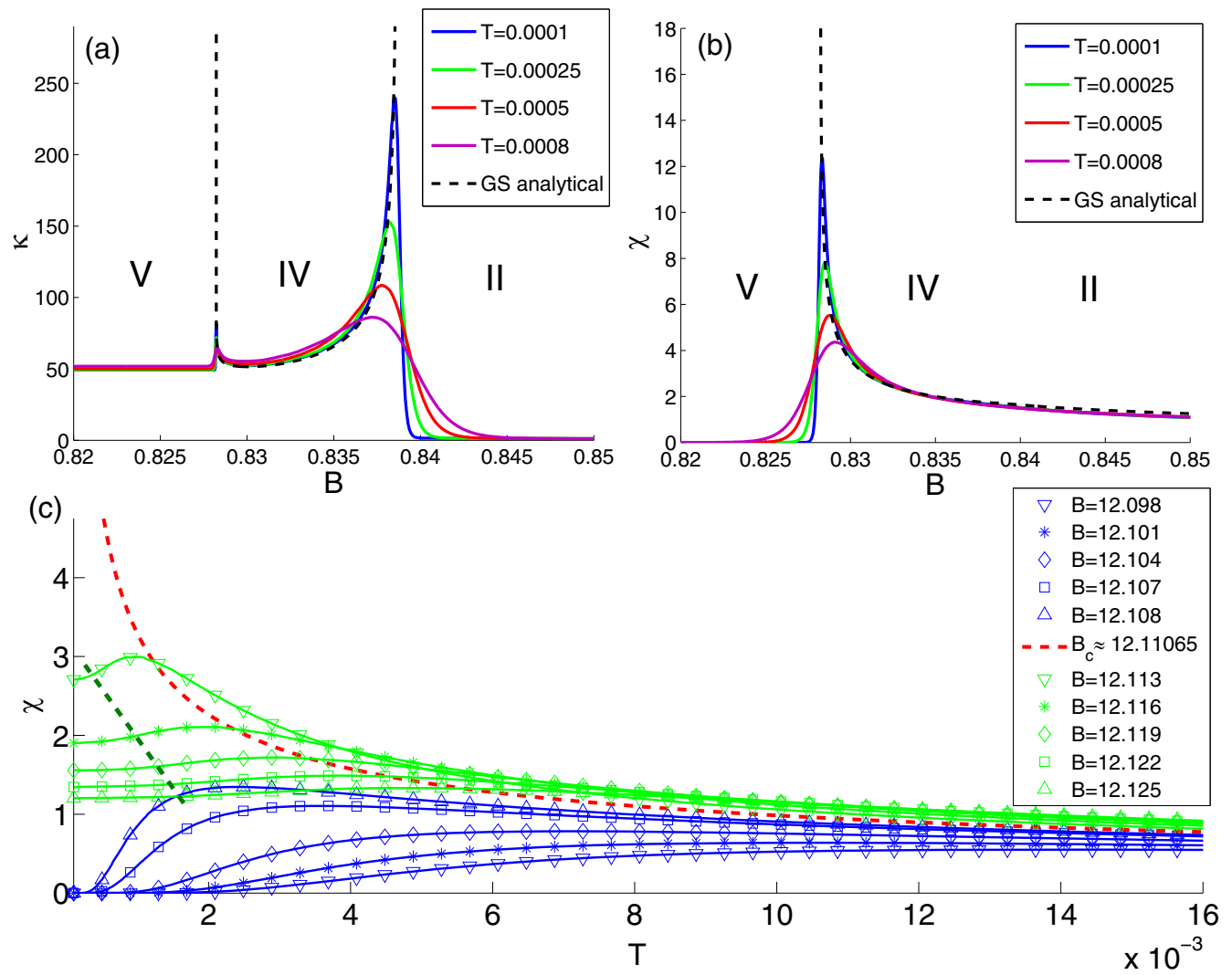

FIG. 3. (a) Compressibility $\kappa$ and (b) spin susceptibility $\chi$ vs magnetic field $B$ for the attractive Hubbard model with $u=-1$ and $\mu=$ -0.8282 . The black dashed lines show the result obtained from the additivity rules (14) with the relations (15) in phase IV. All compressibility and susceptibility curves at low temperature merge into their zero temperature ones given by the additivity rules (14). (c) The red dashed line shows the susceptibility at the critical magnetic field $B_{c}$. For $B<B_{c}$, the susceptibility shows an exponential decay with the energy gap (16). For $B>B_{c}$, the susceptibility is almost temperature independent for the gapless phase, see the region left of the green dashed line. Here the parameters are $u=-7$ and $\mu=-0.08$ for the strong-coupling regime.

by $C_{v}=\frac{\pi T}{3}\left(\frac{1}{v_{u}}+\frac{1}{v_{b}}\right)$. Here, the sound velocities $v_{b, u}$ are as given above.

A second-order phase transition occurs when the system is driven across the phase boundary in the $\mu-B$ plane, see Fig. 1. Figures 3(a) and 3(b) show the compressibility and susceptibility versus magnetic field at different temperatures. They are temperature independent in phase IV, whereas the specific heat depends linearly on the temperature, having thus a common feature of the Fermi liquid in higher dimensions. We observe that in phase IV the compressibility and susceptibility curves at different temperatures collapse into the zero temperature ones obeying the additivity rules (14). Figure 3(c) shows the susceptibility versus temperature for different magnetic fields. For $B>B_{c}$, the susceptibility displays a flat region in the $\chi-T$ plane, the small region to the left of the green dashed line, indicating the two free fluids. For $B<B_{c}$, the susceptibility illustrates the exponential decay as temperature decreases (blue lines). In this case, the susceptibility is given by $\chi_{s}=\frac{T^{-1 / 2}}{4 \sqrt{\pi}} \mathrm{e}^{-\Delta / T}$ with the energy gap

$$
\Delta=-R^{2}+\frac{4\left(2 \pi-R^{3} / 3\right)}{3|u| \pi^{2}}\left(1+\frac{2|u| \pi \mu}{2 \pi-R^{3} / 3}\right)^{3 / 2}
$$

indicating the behavior of dilute magnons. Here we have denoted $R=\operatorname{Re} \sqrt{\mu+2 u+B+2}$.

In summary, for the attractive Hubbard model, we have analytically calculated the FFLO pair correlation and critical exponents, along with scaling functions of thermal and magnetic properties for which the lattice effect becomes prominent. We have obtained the effective chemical potentials of the bound pairs and of the unpaired fermions and demonstrated the additivity rules of the susceptibility and the compressibility in the FFLO-like state. While we have found that the susceptibility and the compressibility are temperature independent, the specific heat depends linearly on the temperature in this phase. These results provide strong evidence for the existence of two free fluids of bound pairs and of unpaired fermions, which were predicted in expansion dynamics of the FFLO state in 1D [26].

Acknowledgments. The authors S.C. and Y.-C.Y. contributed equally to the calculations in this paper. The authors thank M. Takahashi and R. Hulet for helpful discussions. This work is supported by Key NNSFC Grant No. 11534014, the National Key R\&D Program of China No. 2017YFA0304500, NNSFC Grants No. 11374331 and 11174375, and ARC Discovery Projects DP130102839, DP170104934. 
[1] A. C. Hewson, The Kondo Problem to Heavy Fermions (Cambridge University Press, Cambridge, 1997).

[2] T. Giamarchi, Quantum Physics in One Dimension (Oxford University Press, Oxford, 2004).

[3] Y.-P. Wang, Int. J. Mod. Phys. B 12, 3465 (1998).

[4] J. M. P. Carmelo, P. Horsch, and A. A. Ovchinnikov, Phys. Rev. B 45, 7899 (1992).

[5] Y.-C. Yu, Y.-Y. Chen, H.-Q. Lin, R. A. Röemer, and X.-W. Guan, Phys. Rev. B 94, 195129 (2016).

[6] V. R. Shaginyan, V. A. Stephanovich, K. G. Popov, E. V. Kirichenko, and S. A. Artamonov, Ann. Phys. (Berlin) 528, 483 (2016).

[7] F. H. L. Essler, H. Frahm, F. Göhmann, A. Klümper, and V. E. Korepin, The One-Dimensional Hubbard Model (Cambridge University Press, Cambridge, 2005).

[8] R. A. Hart, P. M. Duarte, T.-L. Yang, X. Liu, T. Paiva, E. Khatami, R. T. Scalettar, N. Trivedi, D. A. Huse, and R. G. Hulet, Nature (London) 519, 211 (2015).

[9] A. Singha, M. Gibertini, B. Karmakar, S. Yuan, M. Polini, G. Vignale, M. I. Katsnelson, A. Pinczuk, L. N. Pfeiffer, K. W. West, and V. Pellegrini, Science 332, 1176 (2011).

[10] M. F. Parsons, A. Mazurenko, C. S. Chiu, G. Ji, D. Greif, and M. Greiner, Science 353, 1253 (2016).

[11] R. Zhang, Y. Cheng, H. Zhai, and P. Zhang, Phys. Rev. Lett. 115, 135301 (2015).

[12] L. W. Cheuk, M. A. Nichols, K. R. Lawrence, M. Okan, H. Zhang, E. Khatami, N. Trivedi, T. Paiva, M. Rigol, and M. W. Zwierlein, Science 353, 1260 (2016).

[13] M. Boll, T. A. Hilker, G. Salomon, A. Omran, J. Nespolo, L. Pollet, I. Bloch, and C. Gross, Science 353, 1257 (2016).

[14] V. Ya. Krivnov and A. A. Ovchinnikov, Zh. Eksp. Teor. Fiz. 67, 1568 (1974) [Sov. Phys. JETP 40, 781 (1974)].

[15] N. M. Bogoliubov and V. E. Korepin, Int. J. Mod. Phys. B 3, 427 (1989).

[16] F. Woynarovich and K. Penc, Z. Phys. B 85, 269 (1991).

[17] K.-J.-B. Lee and P. Schlottmann, Phys. Rev. B 38, 11566 (1988).

[18] F. Woynarovich, J. Phys. C 16, 6593 (1983).

[19] P. D. Sacramento, J. Phys.: Condens. Matter 7, 143 (1995).

[20] F. H. L. Essler and V. E. Korepin, Nucl. Phys. B 426, 505 (1994).

[21] P. Fulde and R. A. Ferrell, Phys. Rev. 135, A550 (1964).

[22] A. I. Larkin and Y. N. Ovchinnikov, Zh. Eksp. Teor. Fiz. 47, 1136 (1964). [Sov. Phys. JETP 20, 762 (1965)].

[23] K. Yang, Phys. Rev. B 63, 140511(R) (2001).

[24] M. Tezuka and M. Ueda, Phys. Rev. Lett. 100, 110403 (2008).

[25] A. E. Feiguin and F. Heidrich-Meisner, Phys. Rev. B 76, 220508(R) (2007).
[26] J. Kajala, F. Massel, and P. Törmä, Phys. Rev. A 84, 041601(R) (2011).

[27] M. Gaudin, Phys. Lett. A 24, 55 (1967).

[28] C. N. Yang, Phys. Rev. Lett. 19, 1312 (1967).

[29] X.-W. Guan, M. T. Batchelor, and C.-H. Lee, Rev. Mod. Phys. 85, 1633 (2013).

[30] G. Orso, Phys. Rev. Lett. 98, 070402 (2007).

[31] H. Hu, X.-J. Liu, and P. D. Drummond, Phys. Rev. Lett. 98, 070403 (2007).

[32] Y. Liao, A. S. C. Rittner, T. Paprotta, W. Li, G. B. Partridge, R. G. Hulet, S. K. Baur, and E. J. Mueller, Nature (London) 467, 567 (2010).

[33] S. Cheng, Y.-C. Yu, M. T. Batchelor, and X.-W. Guan, Phys. Rev. B 97, 125145 (2018).

[34] S. Cheng, Y.-Z. Jiang, Y.-C. Yu, M. T. Batchelor, and X.-W. Guan, Nucl. Phys. B 929, 353 (2018).

[35] E. H. Lieb and F. Y. Wu, Phys. Rev. Lett. 20, 1445 (1968).

[36] H. A. Bethe, Z. Phys. 71, 205 (1931).

[37] M. Takahashi, Prog. Theor. Phys. 47, 69 (1972).

[38] M. Takahashi, Prog. Theor. Phys. 52, 103 (1974).

[39] M. Takahashi, Thermodynamics of One-Dimensional Solvable Models (Cambridge University Press, Cambridge, 2005).

[40] Private communication with Professor M Takahashi. If the effective mass of the $k-\Lambda$ strings is positive, they do not form bound states. The effective mass is given by $d^{2} E(q) / d q^{2}$. In the Hubbard model, the mass can be negative at high energy or momentum. Therefore $k-\Lambda$ string bound states can exist at high-energy scales.

[41] We observe that a small value of $\Lambda$ makes a realistic contribution to the free energy of the pairs in the dilute limit. Therefore, for low density, only the leading order of $\xi_{n}(\Lambda)$, i.e., the $\beta_{n}$, is used for the free energy of the pairs.

[42] P. Coleman, Introduction to Many-Body Physics (Cambridge University Press, Cambridge, 2016).

[43] N. M. Bogolyubov and V. E. Korepin, Teor. Mat. Fiz. 82, 231 (1990).

[44] H. Frahm and V. E. Korepin, Phys. Rev. B 42, 10553 (1990).

[45] H. Frahm and V. E. Korepin, Phys. Rev. B 43, 5653 (1991).

[46] Here the asymptotic behavior means the long-distance behavior, i.e., the distance $x$ is large.

[47] J. Y. Lee and X.-W. Guan, Nucl. Phys. B 853, 125 (2011).

[48] X.-W. Guan, M. T. Batchelor, C. Lee, and M. Bortz, Phys. Rev. B 76, 085120 (2007).

[49] I. Boettcher, L. Bayha, D. Kedar, P. A. Murthy, M. Neidig, M. G. Ries, A. N. Wenz, G. Zürn, S. Jochim, and T. Enss, Phys. Rev. Lett. 116, 045303 (2016). 